\title{
Dimensional enhancement of kinetic energies
}

\author{
Schleich, W.P.; Dahl, Jens Peder
}

Published in:

Physical Review A

Link to article, DOI:

10.1103/PhysRevA.65.052109

Publication date:

2002

Document Version

Publisher's PDF, also known as Version of record

Link back to DTU Orbit

Citation (APA):

Schleich, W. P., \& Dahl, J. P. (2002). Dimensional enhancement of kinetic energies. Physical Review A, 65(5), 052109. https://doi.org/10.1103/PhysRevA.65.052109

\section{General rights}

Copyright and moral rights for the publications made accessible in the public portal are retained by the authors and/or other copyright owners and it is a condition of accessing publications that users recognise and abide by the legal requirements associated with these rights.

- Users may download and print one copy of any publication from the public portal for the purpose of private study or research.

- You may not further distribute the material or use it for any profit-making activity or commercial gain

- You may freely distribute the URL identifying the publication in the public portal

If you believe that this document breaches copyright please contact us providing details, and we will remove access to the work immediately and investigate your claim 


\title{
Dimensional enhancement of kinetic energies
}

\author{
W. P. Schleich ${ }^{1}$ and J. P. Dahl $1^{1,2, *}$ \\ ${ }^{1}$ Abteilung für Quantenphysik, Universität Ulm, D-89069 Ulm, Germany \\ ${ }^{2}$ Chemical Physics, Department of Chemistry, Technical University of Denmark, DTU 207, DK-2800 Lyngby, Denmark
}

(Received 27 December 2001; published 18 April 2002)

\begin{abstract}
Simple thermodynamics considers kinetic energy to be an extensive variable which is proportional to the number $N$ of particles. We present a quantum state of $N$ noninteracting particles for which the kinetic energy increases quadratically with $N$. This enhancement effect is tied to the quantum centrifugal potential whose strength is quadratic in the number of dimensions of configuration space.
\end{abstract}

DOI: 10.1103/PhysRevA.65.052109

PACS number(s): 03.65.Yz, 03.75.-b

\section{INTRODUCTION}

The intensity of light radiated from independent dipoles is the number $N$ of dipoles times the intensity of a single one. However, when the dipoles are located within a wavelength of the radiation the total intensity is $N^{2}$ times the intensity of a single dipole [1]. This superradiance effect is due to constructive interference between the individual dipoles. In the present article, we propose an enhancement effect of a similar strength for the kinetic energy of matter waves confined to a region whose radial extension in hyperspace is essentially independent of the number of particles.

According to simple thermodynamics, kinetic energy is an extensive variable, that is, in general the average kinetic energy of an ensemble of $N$ particles is linear in $N$ [2]. A similar dependence holds for the kinetic energy of an ordinary Bose-Einstein condensate (BEC) of $N$ particles. However, we now show that for a special quantum state of $N$ nonrelativistic particles the kinetic energy increases as $N^{2}$. This quantum state is completely symmetric under exchange of the coordinates of the particles, but there is no interaction between the particles.

For the enhancement effect to occur, all particles need to have the same mass, but they do not necessarily have to be identical particles. Nevertheless, the state could also correspond to $N$ identical particles. In this case, it would be the state of $N$ bosons which are strongly entangled.

Dimensional enhancement of kinetic energies is due to the wave nature of the atoms. It results from the form of the Laplacian in $D$ dimensions, giving rise to the quantumcentrifugal potential. We illustrate this phenomenon using $N$ nonrelativistic particles of identical mass $M$ in three space dimensions. Here, we concentrate on the motional degrees of freedom, but do not take into account the internal structure of the particles. Hence, we deal with a $(D=3 N)$-dimensional configuration space, and the wave function $\Psi$ $=\Psi\left(x_{1}, x_{2}, \ldots, x_{D}\right)$ depends on $D$ coordinates.

The proposed effect is most conspicuous for $s$ states, that is, when the wave function depends on the hyperradius, $r$ $=\left(x_{1}^{2}+x_{2}^{2}+\cdots+x_{D}^{2}\right)^{1 / 2}$, only. In this case, the wave function is completely symmetric under exchange of coordinates of the particles, corresponding to a bosonic state. We shall consider only $s$ states in the present work.

Our paper is organized as follows. In Sec. II, we lay the groundwork for the calculation of the kinetic energy of an ensemble of particles, by introducing the concept of the radial wave function in hyperspace. We illustrate it by using two examples related to a BEC in an isotropic harmonic trap. In Sec. III, we then turn to the discussion of the operator of kinetic energy and cast it into a form which brings out the quantum-centrifugal potential. The latter is proportional to the square of the number of dimensions. It is this potential that may cause the kinetic energy to be quadratic in the number of particles, as discussed in Sec. IV.

In Sec. V, we evaluate the kinetic energy of three different radial wave functions. The two motivated by BEC, and denoted by $u_{0}$ and $u_{1}$, show a linear dependence on the number of particles. However, for the third radial wave function $u_{2}$, which is independent of dimensions, we find a kinetic energy that depends on the square of the number of particles. We dedicate Sec. VI to a discussion of the origin of this enhancement effect.

In Sec. VII, we then turn to a discussion of the quantum dynamics, starting from the wave functions $u_{0}, u_{1}$, and $u_{2}$. We evaluate the time dependence of the average radial momentum following from these initial conditions. We note that the momentum corresponding to the BEC wave functions increases with a steepness that is proportional to the square root of the number of particles. In contrast, for the wave function $u_{2}$ we obtain the remarkable result, that the steepness depends quadratically on the number of particles. We conclude in Sec. VIII with a brief summary.

\section{WAVE FUNCTIONS}

For an $s$ state, the motion of the $N$ particles is described by the normalized wave function $\Psi=\Psi(r)$ or, equivalently, by the radial wave function $u(r)$ defined by the relation

$$
\Psi(r)=\frac{1}{\sqrt{S_{D}}} \frac{u(r)}{r^{(D-1) / 2}},
$$

\footnotetext{
*Electronic address: jpd@kemi.dtu.dk
}

where $S_{D}$ denotes the total solid angle in $D$ dimensions, 


$$
S_{D}=\frac{2 \pi^{D / 2}}{\Gamma(D / 2)},
$$

and $u(r)$ is normalized such that

$$
\int_{0}^{\infty}|u(r)|^{2} d r=1
$$

We shall look look at three particular wave functions of the above type. The first one may be constructed from a normalized Gaussian wave function,

$$
\varphi(x)=\left(\frac{\kappa^{2}}{\pi}\right)^{1 / 4} e^{-\kappa^{2} x^{2} / 2}
$$

in one dimension, by forming the product function

$$
\Psi_{0}(r)=\varphi\left(x_{1}\right) \varphi\left(x_{2}\right) \cdots \varphi\left(x_{D}\right)=\left(\frac{\kappa^{2}}{\pi}\right)^{D / 4} e^{-\kappa^{2} r^{2} / 2},
$$

with $r$ being the hyperradius. This wave function describes the state of a BEC of $N=D / 3$ noninteracting particles in an isotropic magnetic trap at zero temperature [3]. The value of $\kappa$ is determined by the harmonic potential of the trap.

Our second $N$-particle wave function, $\Psi_{1}(r)$, may be constructed by retaining the wave function $\varphi(x)$ for $D-1$ space directions, while taking a wave function of the form $x^{2} \varphi(x)$ for the last direction. Symmetrization by forming the coherent sum $\sum_{i=1}^{D} x_{i}^{2} \exp \left(-\frac{1}{2} \kappa^{2} r^{2}\right)$ and subsequent normalization gives in fact

$$
\Psi_{1}(r)=\frac{2 \kappa^{(D+4) / 2}}{\pi^{D / 4} \sqrt{D(D+2)}} r^{2} e^{-\kappa^{2} r^{2} / 2}
$$

The radial wave functions associated with $\Psi_{0}(r)$ and $\Psi_{1}(r)$ are

$$
\begin{aligned}
& u_{0}(r)=\mathcal{N}_{0} r^{(D-1) / 2} e^{-\kappa^{2} r^{2} / 2}, \\
& u_{1}(r)=\mathcal{N}_{1} r^{(D+3) / 2} e^{-\kappa^{2} r^{2} / 2},
\end{aligned}
$$

with the normalization factors $\mathcal{N}_{0}$ and $\mathcal{N}_{1}$ being

$$
\mathcal{N}_{0}=\left[\frac{2}{\Gamma(D / 2)}\right]^{1 / 2} \kappa^{D / 2}, \quad \mathcal{N}_{1}=\left[\frac{2}{\Gamma(D / 2+2)}\right]^{1 / 2} \kappa^{D / 2+2}
$$

We defer the presentation of the third $N$-particle wave function to Sec. V B.

\section{OPERATOR OF KINETIC ENERGY}

We now consider the kinetic energies corresponding to the two above wave functions. The kinetic-energy operator

$$
\hat{T}=-\frac{\hbar^{2}}{2 M} \Delta^{(D)}
$$

is determined by the Laplacian [4]

$$
\Delta^{(D)}=\frac{\partial^{2}}{\partial r^{2}}+\frac{D-1}{r} \frac{\partial}{\partial r}+\frac{\hat{\Lambda}^{2}}{r^{2}}
$$

in $D$ dimensions. The operator $\hat{\Lambda}^{2}$, which involves derivatives with respect to the $D-1$ angles of hyperspace, is proportional to the angular momentum operator in configuration space [5].

As discussed in Ref. [6], we may also write

$$
\hat{T}=\frac{\hat{p}_{r}^{2}}{2 M}+V_{Q}(r)-\frac{\hbar^{2}}{2 M} \frac{\hat{\Lambda}^{2}}{r^{2}} .
$$

Here, we have introduced the radial momentum

$$
\hat{p}_{r}=\frac{1}{2}\left(\frac{\boldsymbol{r}}{r} \cdot \hat{\boldsymbol{p}}+\hat{\boldsymbol{p}} \cdot \frac{\boldsymbol{r}}{r}\right)=\frac{\hbar}{i} \frac{1}{r^{(D-1) / 2}} \frac{\partial}{\partial r} r^{(D-1) / 2},
$$

and the quantum fictitious potential

$$
V_{Q}(r)=\frac{\hbar^{2}}{2 M} \frac{(D-1)(D-3)}{4 r^{2}}
$$

We emphasize that the quantum fictitious potential also emerges directly from acting with the $D$-dimensional Laplacian of Eq. (10) on the wave function $\Psi(r)$ of Eq. (1). This yields, in fact,

$\Delta^{(D)} \Psi=\frac{1}{\sqrt{S_{D}}} \frac{1}{r^{(D-1) / 2}}\left[-\frac{\partial^{2}}{\partial r^{2}}+\frac{(D-1)(D-3)}{4 r^{2}}\right] u(r)$.

Since $V_{Q}$ is proportional to the square of Planck's constant, it is a quantum potential with no classical analog. Moreover, $V_{Q}$ depends inversely on the square of the hyperradius. This feature reminds us of the classical centrifugal potential, which gives rise to the noninertial centrifugal force. Indeed, for $D \geqslant 4$ the potential $V_{Q}$ given by Eq. (13) is positive and thus corresponds to a repulsive force. This property suggests the name quantum-centrifugal potential. In accordance with this, some authors [7] absorb it into the last term of Eq. (11). This, however, conceals the fact that it is a genuine part of the radial kinetic energy.

We note that for $D=2$ the potential $V_{Q}$ is negative corresponding to an attractive force. This centripetal force is unique to two dimensions and counterintuitive to the classical notion of the centrifugal force always being repulsive. To capture this contradiction, we have coined the phrase quantum-anticentrifugal potential for the potential $V_{Q}$ in the case of $D=2$. In Refs. [8] and [9] we have focused on consequences of this attractive potential. However, in the present work we concentrate on the repulsive case corresponding to $D \geqslant 4$.

We recognize that one and three dimensions are also special: the potential $V_{Q}$ vanishes. 


\section{AVERAGE KINETIC ENERGY}

The average kinetic energy

$$
\langle\hat{T}\rangle=-\frac{\hbar^{2}}{2 M} \int_{0}^{\infty} d r r^{D-1} \int d \Omega_{D} \Psi^{*} \Delta^{(D)} \Psi
$$

of the particles described by $\Psi$ involves the Laplacian Eq. (10) and integrations over the hyperradius $r$ and the solid angle $\Omega_{D}$ in the $D$-dimensional hyperspace. With $\Psi=\Psi(r)$, as in the present work, the angular part of the Laplacian, that is, the operator $\hat{\Lambda}^{2} / r^{2}$, does not contribute to the integral. Hence, the kinetic energy may be said to be purely radial in hyperspace, and therefore also in the state spaces of the individual particles.

When we introduce the radial wave function $u(r)$, the average kinetic energy defined by Eq. (15) takes the form

$$
\langle\hat{T}\rangle \equiv T=T_{r}+T_{V}
$$

Here the contribution

$$
T_{r}=\int_{0}^{\infty} d r u^{*}(r)\left[-\frac{\hbar^{2}}{2 M} \frac{d^{2}}{d r^{2}}\right] u(r)
$$

is the average value of the operator $\hat{p}_{r}^{2} / 2 M$. We shall refer to it as the pararadial kinetic energy.

Furthermore, the contribution

$$
T_{V}=\int_{0}^{\infty} d r V_{Q}(r)|u(r)|^{2}
$$

results from $V_{Q}$

\section{EXAMPLES}

The dimension $D$ of configuration space enters $V_{Q}$ quadratically. Hence, the total kinetic energy resulting from $T_{V}$ could in principle be quadratic in $D$. We recall that in the case of $N$ particles in three space dimensions we deal with a $(D=3 N)$-dimensional configuration space. Consequently, for $N \gg 1$, the strength $S \equiv(D-1)(D-3)=(3 N-1)(3 N$ $-3) \simeq 9 N^{2}$ of the quantum noninertial potential is quadratic in the number of particles. According to Eq. (18), the same thing may hold for the contribution $T_{V}$ to the kinetic energy.

\section{A. Thermodynamic case}

However, this feature strongly depends on the form and, in particular, on the $D$ dependence of the radial wave function $u$. For example, the wave functions $u_{0}$ and $u_{1}$ given by Eqs. (7a) and (7b), respectively, yield the radial kinetic energies

$$
T_{r}^{(0)}=\left[1+\frac{1}{2(D-2)}\right] \epsilon, \quad T_{r}^{(1)}=\left[1+\frac{1}{2(D+2)}\right] \epsilon,
$$

and the quantum fictitious potential energies

$$
\begin{gathered}
T_{V}^{(0)}=\left(\frac{D}{2}-1-\frac{1}{2} \frac{1}{D-2}\right) \epsilon, \\
T_{V}^{(1)}=\left(\frac{D}{2}-3+\frac{15}{2} \frac{1}{D+2}\right) \epsilon .
\end{gathered}
$$

Here, we have introduced the kinetic energy $\epsilon$ $\equiv(\hbar \kappa)^{2} /(2 M)$.

Hence, the total kinetic energies

$$
T^{(0)}=\frac{D}{2} \epsilon, \quad T^{(1)}=\left(\frac{D}{2}-2+\frac{8}{D+2}\right) \epsilon
$$

are linear in $D$. In addition, the expression for $T^{(1)}$ involves correction terms independent of and inversely proportional to $D$.

In the limit of a large number of particles, that is, $D$ $=3 N \gg 1$, we recover the thermodynamic result

$$
T \simeq \frac{1}{2} D \epsilon=\frac{3}{2} N \epsilon
$$

for both states.

It is interesting to note that the linear dependence on $D$ shown in Eq. (21) is due to the contribution $T_{V}$, Eqs. (20a) and (20b), which emerges from the quantum-centrifugal potential. The contribution from the pararadial kinetic energy is, according to Eq. (19), only weakly dependent on $D$.

\section{B. Enhancement case}

We now introduce the wave function

$$
u_{2}(r)=\mathcal{N}_{2} \exp \left[-\frac{1}{2}\left(\frac{\beta}{r}+\kappa r\right)\right]
$$

with the normalization constant

$$
\mathcal{N}_{2}=(\beta \kappa)^{-1 / 4}\left(\frac{\kappa}{2 K_{1}(2 \sqrt{\beta \kappa})}\right)^{1 / 2}
$$

Here,

$$
K_{n}(\zeta)=\frac{1}{2} \int_{0}^{\infty} r^{n} \exp \left[-\frac{\zeta}{2}\left(r+\frac{1}{r}\right)\right] \frac{d r}{r}
$$

denotes the modified Bessel function [10] of order $n$.

In contrast to $u_{0}(r)$ and $u_{1}(r)$, Eqs. (7a) and (7b), the wave function $u_{2}(r)$ is independent of the dimension $D$, that is, independent of the number of particles [11]. As a consequence, the cancellation of one power of $D$ in the contribution $T_{V}$ to the kinetic energy which appears for $u_{0}$ and $u_{1}$ cannot take place for $u_{2}$. Indeed, when we substitute the wave function $u_{2}$ into the definitions Eqs. (17) and (18) of the energies $T_{r}$ and $T_{V}$, and perform the integrations, we arrive at

$$
T_{r}^{(2)}=\frac{1}{2 \sqrt{\beta \kappa}} \frac{K_{2}(2 \sqrt{\beta \kappa})}{K_{1}(2 \sqrt{\beta \kappa})} \epsilon
$$


and

$$
T_{V}^{(2)}=\frac{(D-1)(D-3)}{4 \beta \kappa} \epsilon
$$

The pararadial contribution $T_{r}^{(2)}$ is independent of $D$. In contrast, $T_{V}^{(2)}$ involves $D$ and hence the number $N$ of particles quadratically. This enhancement results from the quantum centrifugal potential and reflects the constraint that as we squeeze more particles into the state we do not alter the radial wave function $u_{2}$. Indeed, $u_{2}$ is independent of $D$, and thus independent of $N$. Forcing additional particles into this state leads to a strong increase in energy.

This situation is, to some extent, analogous to the problem of confining charged particles to a given domain of space. Due to their Coulomb interaction

$$
V_{C}(r)=\frac{q_{1} q_{2}}{r}
$$

two charges $q_{1}$ and $q_{2}$ of the same polarity repel each other. This repulsion is proportional to the product of the charges and inversely proportional to their separation.

In the case of neutral particles, the quantum-centrifugal potential of Eq. (13) is proportional to the product $(D-1)$ $\times(D-3)$ of the dimensions. Hence, the dimension of configuration space plays the role of the charge. However, the analogy between $V_{Q}$ and $V_{C}$ breaks down in the dependence on the separation. Indeed, the hyperradius $r$ enters the denominator in a square, whereas in the Coulomb potential the separation enters only linearly.

\section{ORIGIN OF ENHANCEMENT}

This discussion suggests that we can interpret the energy $T_{V}^{(2)}$ associated with the quantum noninertial potential as localization energy. The localization energy is defined as the energy necessary to localize a quantum particle, with the original energy $T_{r}^{(2)}$, in a domain of hyperspace whose radial extension is essentially independent of $D$. This energy obviously depends on the dimension of the space in which we want to localize the particle, because the volume of the unit sphere in $D$ dimensions decreases strongly for large $D$. Indeed, the expression (2) for $S_{D}$, together with an integration over $r$, easily gives the following expression for the volume:

$$
V_{D}=\frac{2 \pi^{(D / 2)}}{D \Gamma(D / 2)}
$$

of the unit sphere. For $D>5$ this is a decreasing function of $D$.

It is interesting that in two dimensions $T_{V}^{(2)}$ becomes negative. Hence, we do not need to perform work in order to localize the particle in this case, but rather gain energy.

It is instructive to compare this quadratic dependence of the kinetic energy on the number of particles to the same scaling property of the total energy of $N$ fermions in a har- monic trap [12] of frequency $\Omega$. Indeed, when we assume that the fermions occupy the first $N$ states of the trap the total energy reads

$E=\hbar \Omega \sum_{j=0}^{N-1}\left(j+\frac{1}{2}\right)=\frac{\hbar \Omega}{2}[N(N-1)+N]=\frac{N^{2} \hbar \Omega}{2}$.

This simple calculation shows that the $N^{2}$ dependence arises from the fact that the particles are trapped in an external classical potential with a linear energy spectrum. In the case of the $N$ particles described by the wave function of Eq. (23), there is no classical potential present. Here, the $N^{2}$ effect results from the quantum centrifugal potential, and from the fact that the strength $S$ of this potential depends quadratically on the number of dimensions of configuration space.

Nevertheless, there exists a potential $V_{2}=V_{2}(r)$ for which the wave function $u_{2}$, Eq. (23), is an energy eigenstate. Indeed, when we differentiate $u_{2}$ twice with respect to $r$ we find that $u_{2}$ satisfies the time independent Schrödinger equation

$$
\frac{d^{2} u(r)}{d r^{2}}+\frac{2 M}{\hbar^{2}}\left[E-V_{2}(r)\right] u(r)=0
$$

with energy $E=0$ and the potential

$$
V_{2}(r)=\frac{\hbar^{2}}{2 M}\left[\frac{1}{4} \frac{\beta^{2}}{r^{4}}-\frac{\beta \kappa}{2 r^{2}}-\frac{\beta}{r^{3}}+\left(\frac{\kappa}{2}\right)^{2}\right] .
$$

Since $u_{2}$ is independent of $D$, the potential $V_{2}$ also has to be independent of $D$.

We recall from Eq. (17) that $d^{2} u(r) / d r^{2}$ determines the pararadial kinetic energy rather than the total kinetic energy. To get the Schrödinger equation for the wave function $\Psi_{2}(r)$, as related to $u_{2}(r)$ through Eq. (1), we draw on the expressions (11) and (14), and get

$$
-\frac{\hbar^{2}}{2 M} \Delta^{(D)} \Psi_{2}(r)+\left[V_{2}(r)-V_{Q}(r)\right] \Psi_{2}(r)=E \Psi_{2}(r) .
$$

The confining potential in hyperspace is accordingly $V_{2}(r)$ $-V_{Q}(r)$.

\section{QUANTUM DYNAMICS}

So far, we have focused on quantum kinematics. Now we turn to a discussion of the dynamics resulting from the three radial wave functions $u_{0}(r), u_{1}$, and $u_{2}$, considered as initial wave functions when we switch off the confining potentials at time $t=0$, and hence allow for free-particle motion of the systems. Here we concentrate on the case of many particles, that is $D=3 N \gg 3$.

The dynamics of this ensemble of free particles is governed by the Schrödinger equation

$$
i \hbar \frac{\partial}{\partial t} \Psi(r, t)=-\frac{\hbar^{2}}{2 M} \Delta^{(D)} \Psi(r, t) .
$$


By exploiting the relation (14) for $\Psi$ we obtain the following expression for the time evolution of the radial wave function:

$$
i \hbar \frac{\partial}{\partial t} u(r, t)=\left[-\frac{\hbar^{2}}{2 M} \frac{\partial^{2}}{\partial r^{2}}+V_{Q}(r)\right] u(r, t) .
$$

For short times, we may obtain a first approximation to $u(r, t)$ by replacing Eq. (35) with the equation

$$
i \hbar \frac{\partial}{\partial t} u(r, t)=\left[W(r)+V_{Q}(r)\right] u(r, 0),
$$

where

$$
W(r)=\frac{1}{u(r, 0)}\left[-\frac{\hbar^{2}}{2 M} \frac{\partial^{2}}{\partial r^{2}}\right] u(r, 0) .
$$

This quantity is similar to Bohm's quantum potential [13]. Integration gives

$$
u(r, t) \simeq \exp \left\{-\frac{i}{\hbar}\left[W(r)+V_{Q}(r)\right] t\right\} u(r, 0) .
$$

The formula (38) allows us to derive an analytical expression for the short-time behavior of the average radial momentum

$$
\left\langle p_{r}\right\rangle(t)=\int_{0}^{\infty} d r r^{D-1} \int d \Omega_{D} \Psi *(r, t) \hat{p}_{r} \Psi(r, t) .
$$

By means of the definition Eq. (12) of the radial momentum $\hat{p}_{r}$ and the ansatz Eq. (1) for the radial wave function, this expression reduces to

$$
\left\langle p_{r}\right\rangle(t)=\int_{0}^{\infty} d r u^{*}(r, t)\left(\frac{\hbar}{i} \frac{\partial}{\partial r}\right) u(r, t) .
$$

When we substitute the approximate solution Eq. (38) into this formula, we observe that $W(r)$ does not contribute to the integral because $u(r, 0)$ is real valued. Hence, we arrive at

$$
\left\langle p_{r}\right\rangle(t) \simeq \int_{0}^{\infty} d r F_{Q}(r)|u(r, 0)|^{2} t,
$$

where

$$
F_{Q}(r)=-\frac{d V_{Q}}{d r}=\frac{\hbar^{2}}{2 M} \frac{(D-1)(D-3)}{2 r^{3}} .
$$

This quantity, determined by the derivative of the potential $V_{Q}$, is the quantum-centrifugal force. The fact that the expression for $\left\langle p_{r}\right\rangle(t)$ is independent of $W(r)$ implies that we obtain the correct result for the short-time behavior of $\left\langle p_{r}\right\rangle(t)$ by neglecting the pararadial kinetic energy operator compared to the potential $V_{Q}$ in Eq. (35). The result of doing so is known as the Raman-Nath approximation [14]. We also note that the integral preceding $t$ in Eq. (41) is nothing but $d\left\langle p_{r}\right\rangle(t) / d t$ evaluated at $t=0$. The expression (41) may therefore also be obtained by differentiating both sides of Eq. (40) with respect to $t$, while applying Ehrenfest's theorem [15].

To sum up, we have found that the initial average radial momentum increases linearly in time. The slope of the increase is determined by the average centrifugal force of the initial state.

Let us now consider the specific wave functions $u_{0}, u_{1}$, and $u_{2}$. We start our discussion with the wave functions $u_{0}$ and $u_{1}$ of Eqs. (7a) and (7b), respectively. Substitution into the expression (41) for the average radial momentum yields

$$
\begin{gathered}
\left\langle p_{r}\right\rangle^{(0)}(t) \simeq(D-1) \frac{\Gamma((D-1) / 2)}{\Gamma(D / 2)}\left(\frac{\epsilon t}{\hbar}\right) \hbar \kappa, \\
\left\langle p_{r}\right\rangle^{(1)}(t) \simeq \frac{1}{2}(D-1)(D-3) \frac{\Gamma((D+1) / 2)}{\Gamma((D+4) / 2)}\left(\frac{\epsilon t}{\hbar}\right) \hbar \kappa .
\end{gathered}
$$

With the help of the asymptotic formula [10]

$$
\Gamma(a z+b) \sim \sqrt{2 \pi} e^{-a z}(a z)^{a z+b-1 / 2},
$$

we can evaluate the ratios

$$
\frac{\Gamma((D-1) / 2)}{\Gamma(D / 2)} \sim\left(\frac{2}{D}\right)^{1 / 2}, \quad \frac{\Gamma((D+1) / 2)}{\Gamma((D+4) / 2)} \sim\left(\frac{2}{D}\right)^{3 / 2}
$$

in the limit $D \gg 3$. For both wave functions, this yields

$$
\left\langle p_{r}\right\rangle(t) \sim \sqrt{2 D}\left(\frac{\epsilon t}{\hbar}\right) \hbar \kappa
$$

Hence, in the case of the initial wave function $u_{0}$ and $u_{1}$, the slope of the momentum increase is governed by the square root of the number of dimensions [16].

Next, we turn to the wave function $u_{2}$, Eq. (23). In this case, the average radial momentum Eq. (40) takes the form

$$
\left\langle p_{r}\right\rangle(t) \simeq \frac{(D-1)(D-3)}{2(\beta \kappa)^{3 / 2}} \frac{K_{2}(2 \sqrt{\beta \kappa})}{K_{1}(2 \sqrt{\beta \kappa})}\left(\frac{\epsilon t}{\hbar}\right) \hbar \kappa .
$$

Thus, the momentum corresponding to the wave function $u_{2}$ increases as $D^{2}$, and hence depends quadratically on the number of particles.

The explosion of the particles may be compared with the phenomenon of a Coulomb explosion [17]. Molecular bound atoms passing through a foil get stripped of some their electrons and become positively charged ions. Due to their Coulomb interaction, they repel each other and fly apart. In our case of neutral particles, it is the quantum-centrifugal potential $V_{Q}$, Eq. (13), which causes the explosion. 


\section{SUMMARY}

We conclude by summarizing our main results. We have analyzed the kinetic energy and the dynamics of $N$ noninteracting particles in free space. We have found a quantum state for which the average kinetic energy increases quadratically with the number of particles. Moreover, this kinetic energy stored in the state gets transferred into outgoing radial momentum in the time evaluation pursuant to the preparation of the wave function. In the case of $u_{2}$ the explosion is more violent than for $u_{0}$ and $u_{1}$, since the increase of momentum is proportional to $N^{2}$ rather than $N^{1 / 2}$. Both effects-the dimensional enhancement of kinetic energies and the $N^{2}$ explosion-are consequences of the quantum-centrifugal potential.

\section{ACKNOWLEDGMENTS}

We thank G. Alber, E. Arimondo, I. Bialynicki-Birula, F. Bopp, J. Botero, A. Delgado, M. Mussinger, K. Vogel, and W. Wonneberger for many fruitful discussions. J.P.D. gratefully acknowledges the support of the Alexander von Humboldt Stiftung.
[1] R. H. Dicke, Phys. Rev. 93, 99 (1954).

[2] See, for example, R. C. Tolman, Relativity, Thermodynamics, and Cosmology (Clarendon, Oxford, 1934).

[3] See, for example, G. Baym and C. J. Pethick, Phys. Rev. Lett. 76, 6 (1996).

[4] A. Sommerfeld, Partielle Differentialgleichungen der Physik (Geest and Portig, Leipzig, 1947); Partial Differential Equations in Physics (Academic Press, New York, 1949).

[5] J. Avery, Hyperspherical Harmonics. Applications in Quantum Theory (Kluwer, London, 1989).

[6] J. P. Dahl and W. P. Schleich, Phys. Rev. A 65, 022109 (2002).

[7] D. R. Herschbach, in Dimensional Scaling in Chemical Physics, edited by D. R. Herschbach, J. Avery, and O. Goscinski (Kluwer, Dordrecht, 1993), p. 61.

[8] M. A. Cirone, G. Metikas, and P. Schleich, Z. Naturforsch., A: Phys. Sci. 56a, 48 (2001); M. A. Cirone, J. P. Dahl, M. Fedorov, D. Greenberger, and W. P. Schleich, J. Phys. B 35, 191 (2002); M. A. Cirone, K. Rzązweski, W. P. Schleich, F. Straub, and J. A. Wheeler, Phys. Rev. A 65, 022101 (2002).

[9] I. Białynicki-Birula, M. A. Cirone, J. P. Dahl, M. Fedorov, and W. P. Schleich, e-print quant-ph/0110116 (unpublished).

[10] Handbook of Mathematical Functions, Natl. Bur. Stand. Appl.
Math. Ser. No. 55, edited by M. Abramowitz and I. Stegun (U.S. GPO, Washington, DC, 1964).

[11] There are strict conditions on such a radial function. In particular, all its derivatives must vanish at $r=0$ in order that a proper $\Psi(r)$ of the form (1) may be constructed for any $D$. The function $u_{2}(r)$ fulfills this requirement.

[12] See, for example, F. Gleisberg, W. Wonneberger, U. Schlöder, and C. Zimmermann, Phys. Rev. A 62, 063602 (2000).

[13] See, for example, P. R. Holland, The Quantum Theory of Motion (Cambridge University Press, Cambridge, England, 1993).

[14] W. P. Schleich, Quantum Optics in Phase Space (Wiley-VCH, Weinheim, 2001).

[15] L. I. Schiff, Quantum Mechanics, 3rd ed. (Wiley, New York, 1968).

[16] For large values of $t$, the radial momentum settles at a constant value $p_{\infty}$. In the limit $D \gg 3$ this value may be determined from Eq. (22) by writing $T=p_{\infty}^{2} / 2 M$. This gives $p_{\infty}$ $=\sqrt{D / 2} \hbar \kappa$. Hence, we may also write $\left\langle p_{r}\right\rangle(t) \sim 2 p_{\infty}(\epsilon t / \hbar)$. This is in accordance with the results of our discussion of $s$-wave implosion and explosion in Ref. [9].

[17] Z. Vager, R. Naaman, and E. P. Kanter, Science 244, 426 (1989). 